\title{
Survey of Medicinal Plants in Mahadevan Hill, K.V. Kuppam, At Vellore District, Tamil Nadu, India
}

\author{
D. Devendiran, H. Abdul Jaffar Ali, K. Muhammed Shariq and M.A. Farook* \\ Department of Biotechnology, Islamiah College (Autonomous), Vaniyambadi-635 752, India \\ *Corresponding author
}

A B S T R A C T

\begin{tabular}{l} 
Ke y w or d s \\
$\begin{array}{l}\text { Medicinal plant, } \\
\text { traditional } \\
\text { medicines, diseases, } \\
\text { Mahadevan hill, } \\
\text { flora and } \\
\text { biodiversity }\end{array}$ \\
\hline Article Info \\
$\begin{array}{l}\text { Accepted: } \\
\text { 05 February } 2020 \\
\text { Available Online: } \\
\text { 10 March 2020 }\end{array}$ \\
\hline
\end{tabular}

In order to document the medicinal plants and their applications, a field survey was undertaken at Mahadeva hill which is located $28 \mathrm{Km}$ away from Vellore city and $60 \mathrm{k} . \mathrm{m}$. from Islamiah College (Autonomous), Vaniyambadi, TamilNadu, India. An attempt has been made to identify the medicinal plants traditionally used by the people of the region and also to preserve the traditional heritage. Further this study highlights the risks of extensive human exploitation of biodiversity of the region. The survey was carried out during January to November 2019. A total of 88 herbaceous medicinal plants were documented and an inventory was prepared. All these 88 plants species are belonging to 40 families such as Acanthaceae, Aizoaceae, Alangiaceae, Alliaceae, Amaranthaceae, Annonaceae, Apocyanaceae, Aristolochiaceae, Asclepiadaceae, Asteraceae, Bombacaceae, Caesalpinacea, Capparaceae, Caricaceae, Cesalpinioideae, Cleomaceae, Cucurbitaceae, Euphorbiaceae, Fabaceae, Gentianaceae, Labiatae, Lamiaceae, Liliaceae, Lythraceae, Malvaceae, Meliaceae, Mimosaceae, Moraceae, Myrtaceae, Oleaceae, Oxalidaceae, Papilionoideae, Poaceae, Rhamnaceae, Rubiaceae, Rutaceae, Sapindaceae, Solanaceae, Verbanaceae and Vitaceae.This quantitative survey gives an account on the floral diversity and uses of medicinal plants.

\section{Introduction}

India has contributes eight percent of total world bio-resources and represents one among the 12 mega-diversity countries in the world due to the species richness and level of endemism recorded in the various agro climatic areas of the country. There are about 17,000 species of higher plants, of which approximately 8,000 species are considered medicinal plants and used by village communities, particularly tribal communities, or in traditional medicinal systems, such as the Ayurveda.

Since the start of human civilization, medicinal plants have been used by mankind for its therapeutic importance. The term medicinal plants include a various types of plants used in herbalism and some of these 
plants have a medicinal activities. Medicinal plants are the "backbone" of traditional medicine, which means more than 3.3 billion people in the underdeveloped countries utilize medicinal plants on a regular basis (Davidson-Hunt2000). These medicinal plants are considered as rich resources of constituents which can be used in drug development and synthesis. Besides, these plants play a acute role in the development of human cultures around the whole world.

Current evaluations suggest that, in many developing countries, a large proportion of the population relies heavily on traditional practitioners and medicinal plants to meet primary health care needs. Although recent medicine may be available in these countries, herbal medicines have often maintained popularity for historical and cultural reasons. Medicinal plants are frequently used as raw materials for extraction of active ingredients and used in the synthesis of different drugs. Asin case of laxatives, blood thinners, antibiotics and anti-malarial medications, contain plants ingredients. Moreover, the active constituents of Taxol, vincristine, and morphine isolated from foxglove, periwinkle, yew, and opium poppy respectively.

The development and commercialization of medicinal plant based bio-industries in the developing countries is dependent upon the availability of facilities and information concerning upstream and downstream bioprocessing, extraction, purification, and promoting of the industrial prospective of medicinal plants.

Furthermore, the absence of efficient socioeconomic and public healthcare systems reinforces reliance of rural and lower-income urban populations on the use of traditional medicinal herbs and plants as complementary aids to routine pharmaceutical market products. A recent estimate suggests that nearly 9,000 plants have known medicinal applications in various cultures and countries, and this is without having conducted comprehensive research amongst several indigenous and other communities. The Ethnobotanical studies of different tribal localities may lead to find new information on unexploited natural resources as sources of medicine, food, fodder, fibre, and animal habitat.

The Mahadevan hill is a very rich forested area in terms of plants, Animal and flower diversity which provide many valuable medicinal plants and tribals of the study area are using these plants since ancient time which is yet to record in a proper scientific way. The detailed information on medicinal uses of plants is not available or not recorded well. So the present study suggests that to document all the plant biodiversity resources for the first time.

\section{Materials and Methods}

\section{Description of study area}

Mahadevan hills in Kanguppam in Vellore district of Tamil Nadu State, India. It is located $25 \mathrm{KM}$ towards west from District headquarters Vellore. $9 \mathrm{Km}$ from K.V.Kuppam and $163 \mathrm{Km}$ from State capital Chennai.

\section{Methodology}

The study area was surveyed randomly from January to November 2019. Data was collected from the local People and medicinal practitioners inhabiting in and around the Mahadevan hill. In this study, data collection was carried out through interviews and detailed personal discussions with the local people who are unique in usage of medicinal plants. The discussion contained the details of the plants, parts used, medicinal uses and mode of preparation. 


\section{Results and Discussion}

The Study was based on the survey of traditional information on the medicinal plants from Mahadevan hill of K.V. Kuppam, Vellore district. In this study, we engaged mainly on plant species reported by the native people in and around the study region for their medicinal practices. Present data are the general results of the ethnobotanical survey conducted from January 2019 to November 2019. Herbal therapies are considered the ancient forms of health care known to mankind on this world.

Prior to the development of recent medicine, the traditional classifications of medicine that has advanced over the centuries within various communities, are still maintained as a great traditional information base onherbal medicines (Mukherjee and Wahil, 2006). Traditionally, this wealth of precious knowledge has been passed on orally from generation to generation without any written document and is still retained by various indigenous groups around the world (Perumal Samy and Ignacimuthu, 2000 and Saranraj et al., 2016).

Due to the growth of demand for biochemical diversity in screening programs, seeking therapeutic remedies from natural products, interest particularly in ethno medicinal plants has grown throughout the world (Saranraj and Sujitha, 2015). Herbal therapies are considered as the oldest forms of health care known to mankind on this earth. Traditional popular medicine uses the knowledge, skills and practices based on the concepts, beliefs and experiences indigenous to its cultures for maintenance of health.

Documenting the native knowledge through ethnobotanical studies is vital for the conservation and utilisation of biological resources. Ethnobotanical survey has been found to be one of the consistent approaches to medicine discovery (Fabricant and Farnsworth, 2001; Kolanjinathan and Saranraj, 2015).

Traditional uses of 88 plant species spread over 40 families are described under this study. The tribes and peoples from various rural villages have used the medicinal plants to treat various diseases and disorders such asasthma, bronchitis, cough, diabetes, fever, gonorrhoea, heart troubles, intestinal worms, jaundice, leprosy, malaria, snakebites, sore eyes, toothache, tuberculosis, wounds etc.The medicinal plants used by them are arranged by scientific name with Family name, Vernacular name, Plant parts used and Therapeutic uses in Table-1

The plants documented in this survey belong to the families such as Acanthaceae, Aizoaceae, Alangiaceae, Alliaceae, Amaranthaceae, Annonaceae, Apocyanaceae, Aristolochiaceae, Asclepiadaceae, Asteraceae, Bombacaceae, Caesalpinacea, Capparaceae, Caricaceae, Cesalpinioideae, Cleomaceae, Cucurbitaceae, Euphorbiaceae, Fabaceae, Gentianaceae, Labiatae, Lamiaceae, Liliaceae, Lythraceae, Malvaceae, Meliaceae, Mimosaceae, Moraceae, Myrtaceae, Oleaceae, Oxalidaceae, Papilionoideae, Poaceae, Rhamnaceae, Rubiaceae, Rutaceae, Sapindaceae, Solanaceae, Verbanaceae and Vitaceae.

In India, there are about 54 million native people of different traditional groups inhabiting various landscapes. These native groups possess their own distinct culture, religious rites, food habit and have a rich knowledge of traditional treatment. Even today, native and certain local communities practiced herbal treatment to cure a range of diseases, with plants particularly used as popular medicine to treat snakebites (Parinitha et al., 2005). 
Consumption of plants for medicinal purposes in India has been documented long back in olden literature because they are necessary to human survival (Mohamed Tariq and $\mathrm{Md}$ Rayees Ifham, 2013; Sastri et al., 1996). The consumption, management and estimation of wild plants are central aspects of the traditional knowledge in many human populations. Thus, plants gathering, the distribution and conservation of knowledge within the community are traditional performs that have contribution to the survival of many cultures. In most of the cultures the medical system coexists with several traditional systems. These traditional medical systems are generally based on the uses of natural and native products which are normally related to the people's perspective on the world and life (Toledo et al., 2009). In this study, a botanical survey was carried out in Vellore district, Tamilnadu, India.

Table.1 List of Medicinal Plants in Mahadevan Hill, K.V. Kuppam, Vellore District

\begin{tabular}{|c|c|c|c|c|}
\hline S. No. & Plant Name & Local Name & Parts Used & Therapeutic Use \\
\hline 1 & $\begin{array}{l}\text { Abutilon indicum } \\
\text { (Malvaceae) }\end{array}$ & Thuthi & Leaf & Antimicrobial activity. \\
\hline 2 & $\begin{array}{l}\text { Acalypha indica } \\
\text { (Euphorbiaceae) }\end{array}$ & Kuppaimeni & Leaf & $\begin{array}{l}\text { Leaf juice is applied externally } \\
\text { for curing body itching. }\end{array}$ \\
\hline 3 & $\begin{array}{l}\text { Achyranthes aspera } \\
\text { (Amaranthaceae) }\end{array}$ & Naivooruvi & Leaf & Wounds \\
\hline 4 & $\begin{array}{l}\text { Acmella oleracea } \\
\text { (Asteraceae) }\end{array}$ & Therupallupoondu & $\begin{array}{l}\text { leaves and } \\
\text { flowers }\end{array}$ & $\begin{array}{l}\text { Stammering, toothache, } \\
\text { and stomatitis }\end{array}$ \\
\hline 5 & $\begin{array}{l}\text { Adhatoda vasica } \\
\text { (Acanthaceae) }\end{array}$ & Adathodai & Aerial parts & $\begin{array}{c}\text { Bronchitis, Leprosy, Heart } \\
\text { troubles, Asthma, Cough, Sore } \\
\text { eyes and Gonorrhea. }\end{array}$ \\
\hline 6 & $\begin{array}{l}\text { Aeglemarmelos } \\
\text { (Rutaceae) }\end{array}$ & Vilvam & Leaf & $\begin{array}{c}\text { The dried and powdered leaves } \\
\text { are used for diabetes. }\end{array}$ \\
\hline 7 & $\begin{array}{c}\text { Aervalanata } \\
((\text { Amaranthaceae }))\end{array}$ & Sirukanpeelai & Wholeplant & Snakebites \\
\hline 8 & $\begin{array}{c}\text { Allium сера } \\
\text { (Alliaceae) }\end{array}$ & Vengkaayam & Bulb onion & $\begin{array}{c}\text { Bulb of the onion is used for } \\
\text { diabetes. }\end{array}$ \\
\hline 9 & $\begin{array}{l}\text { Alangium salvifolium } \\
\text { (Alangiaceae) }\end{array}$ & Alengi & Aerial part & $\begin{array}{l}\text { Antioxidant and antimicrobial } \\
\text { activities. }\end{array}$ \\
\hline 10 & $\begin{array}{c}\text { Aloe vera } \\
\text { (Liliaceae) }\end{array}$ & Kathazai & Aerial parts & $\begin{array}{c}\text { Promotes menstrual flow, Heals } \\
\text { wounds and fresh cuts, Eye } \\
\text { diseases, Asthma, Leprosy and } \\
\text { Jaundice. }\end{array}$ \\
\hline 11 & $\begin{array}{l}\text { Amaranthus virudis } L . \\
\text { (Amaranthaceae) }\end{array}$ & Thoia & Leaf & $\begin{array}{l}\text { Leaf paste is applied over the } \\
\text { infected area to cure erysipelas. }\end{array}$ \\
\hline 12 & $\begin{array}{c}\text { Anisomeles malabarica } \\
\text { (Labiatae) }\end{array}$ & Peruntumpai & Aerial part & $\begin{array}{l}\text { The plant is useful halitosis, } \\
\text { amentia, intestinal worms and } \\
\text { fever arising from teething in } \\
\text { children. }\end{array}$ \\
\hline 13 & $\begin{array}{c}\text { Annona squamosal } \\
\text { (Annonaceae) }\end{array}$ & Setha & Fruits & Intestinal worms. \\
\hline 14 & $\begin{array}{l}\text { Aristolochia bracteolate } \\
\text { (Aristolochiaceae) }\end{array}$ & Aaduthinnappalai & Leaf & $\begin{array}{l}\text { Leaf juice is taken orally to } \\
\text { treatment of diabetes. }\end{array}$ \\
\hline
\end{tabular}




\begin{tabular}{|c|c|c|c|c|}
\hline 15 & $\begin{array}{l}\text { Artocarpus hirsutus Lam. } \\
\text { (Moraceae) }\end{array}$ & Kattupala & Fruit & $\begin{array}{l}\text { Fruits used as appetizer, seed } \\
\text { with honey used to treat asthma. }\end{array}$ \\
\hline 16 & $\begin{array}{c}\text { Azadirachta indica } \\
\text { (Meliaceae) }\end{array}$ & Veempu (Neem) & Leaf & $\begin{array}{c}\text { Powdered leaves are used for } \\
\text { diabetes. }\end{array}$ \\
\hline 17 & $\begin{array}{c}\text { Blepharis medaraspatensis }(L) \\
\text { (Acanthaceae) }\end{array}$ & Elumbuotti & Seeds & $\begin{array}{l}\text { dysuria, diseases of nervous } \\
\text { system, diuretic, aphrodisiac }\end{array}$ \\
\hline 18 & $\begin{array}{c}\text { Cajanus cajan } \\
\text { (Fabaceae) }\end{array}$ & Thovaray & Seed & $\begin{array}{l}\text { Seeds boiled and taken along } \\
\text { with food items }\end{array}$ \\
\hline 19 & $\begin{array}{l}\text { Calotropis gigantean } \\
\text { (Asclepiadaceae) }\end{array}$ & Erukkam & Leaf Milk & $\begin{array}{l}\text { Cure rheumatic Joints pain and } \\
\text { swellings. }\end{array}$ \\
\hline 20 & $\begin{array}{l}\text { Cardiospermum } \\
\text { halicacabum } \\
\text { (Sapindaceae) }\end{array}$ & Mudakkaththan & Leaf & Rheumatic arthritis \\
\hline 21 & $\begin{array}{l}\text { Carica papaya } \\
\text { (Caricaceae) }\end{array}$ & Pappali & Leaf, fruits & $\begin{array}{l}\text { Tuberculosis and promotes } \\
\text { menstrual flow. }\end{array}$ \\
\hline 22 & $\begin{array}{c}\text { Cassia alata } \\
\text { (Cesalpinioideae) }\end{array}$ & Seemaiakathi & Leaf & $\begin{array}{c}\text { Ringworm patches, cough and } \\
\text { eczema. }\end{array}$ \\
\hline 23 & $\begin{array}{l}\text { Cassia auriculata } \\
\text { (Caesalpinacea) }\end{array}$ & Avaram & Flower & $\begin{array}{c}\text { Daily three or four flower are } \\
\text { taken regularly }\end{array}$ \\
\hline 24 & $\begin{array}{c}\text { Cassia fistula } \\
\text { (Cesalpinioideae) }\end{array}$ & Sarakondrai, Konnei & Aerial part & $\begin{array}{l}\text { Bark decoction mixed with } \\
\text { garlic and powdered pepper is } \\
\text { given to cattle as purgative. }\end{array}$ \\
\hline 25 & $\begin{array}{c}\text { Cassia obtuse } \\
\text { (Cesalpinioideae) }\end{array}$ & Nilaavarai & Leaf & Antimicrobial activity. \\
\hline 26 & $\begin{array}{l}\text { Cassia roxburghii } \\
\text { (Cesalpinioideae) }\end{array}$ & Sennkondrai & Leaf & Hepatoprotective activity. \\
\hline 27 & $\begin{array}{c}\text { Cassia tora } \\
\text { (Cesalpinioideae) }\end{array}$ & Tagarai & Aerial part & $\begin{array}{l}\text { Malaria, ring worm, chronic } \\
\text { inflammation of the skin and } \\
\text { other skin diseases. }\end{array}$ \\
\hline 28 & $\begin{array}{l}\text { Catharanthus roseus } \\
\text { (Apocyanaceae) }\end{array}$ & Nithyakalyani & Aerial parts & $\begin{array}{l}\text { Antimicrobial and Anticancer } \\
\text { activity. }\end{array}$ \\
\hline 29 & $\begin{array}{l}\text { Ceibapentandra } \\
\text { (Bombacaceae) }\end{array}$ & Ilavam & Aerial part & $\begin{array}{l}\text { Diuretic, antipyretic, tonic, } \\
\text { gonorrhoea, dysuria, acrid, } \\
\text { bitter, thermogenic febrifuge, } \\
\text { emetic tonic and tumours. }\end{array}$ \\
\hline 30 & $\begin{array}{l}\text { Celosia argentea } \\
\text { (Amaranthaceae) }\end{array}$ & Pannaikeerai & Leaf & Antimicrobial activity. \\
\hline 31 & $\begin{array}{c}\text { Cissusquadrangularis } \\
\text { (Vitaceae) }\end{array}$ & Perandai & $\begin{array}{l}\text { Aerial } \\
\text { Parts }\end{array}$ & $\begin{array}{l}\text { Heart diseases, diabetes and } \\
\text { metabolic Syndrome }\end{array}$ \\
\hline 32 & $\begin{array}{c}\text { Citrus limon (Linn.) Burm. } \\
\text { (Rutaceae) }\end{array}$ & Elumichai & Fruit & $\begin{array}{l}\text { Fruit is used to cure nail } \\
\text { infection, juice induces } \\
\text { freshness to body. }\end{array}$ \\
\hline 33 & $\begin{array}{l}\text { Cleome viscosa } \\
\text { (Cleomaceae) }\end{array}$ & Naaikadugu & Leaves & $\begin{array}{c}\text { Wounds, ulcers, discharge of } \\
\text { pus from the ear. }\end{array}$ \\
\hline 34 & $\begin{array}{l}\text { Clitoria ternatea } \\
\text { (Fabaceae) }\end{array}$ & SanguPushpam & Leaf & $\begin{array}{l}\text { Antimicrobial activity, Eye } \\
\text { diseases and Headache. }\end{array}$ \\
\hline 35 & $\begin{array}{l}\text { Coccinia grandis } \\
\text { (Cucurbitaceae) }\end{array}$ & Kovai & Leaf & Eye diseases \\
\hline 36 & Couroupita guianensis & Naga Lingam & several parts & Hypertension, tumors, pain, \\
\hline
\end{tabular}




\begin{tabular}{|c|c|c|c|c|}
\hline & (Amaranthaceae) & & of the tree & $\begin{array}{l}\text { inflammation, common } \\
\text { cold, stomachache, skin } \\
\text { conditions, wounds, malaria, } \\
\text { and toothache. }\end{array}$ \\
\hline 37 & $\begin{array}{l}\text { Crateva adansonii } \\
\text { (Capparaceae) }\end{array}$ & Mavelangam & Leaf & Fever, acrid and cough \\
\hline 38 & $\begin{array}{l}\text { Cucumis sativus } \\
\text { (Cucurbitaceae) }\end{array}$ & Vellari & $\begin{array}{l}\text { Leaf } \\
\text { Fruits }\end{array}$ & $\begin{array}{c}\text { Fever, Bronchitis, Jaundice, } \\
\text { HaemorrphagesStrangury and } \\
\text { General debility. }\end{array}$ \\
\hline 39 & $\begin{array}{l}\text { Cynodon dactylon } \\
\text { (Poaceae) }\end{array}$ & Arugampul & $\begin{array}{l}\text { Whole } \\
\text { parts }\end{array}$ & Antimicrobial and Diabetes. \\
\hline 40 & $\begin{array}{l}\text { Datura metal } \\
\text { (Solanaceae) }\end{array}$ & Oomatthai & Leaves & $\begin{array}{l}\text { Asthma, cough, tuberculosis, } \\
\text { bronchitis, haemorrhoids, boils, } \\
\text { sores, skin diseases, } \\
\text { rheumatism, headache, } \\
\text { toothache, cholera, abortifacient } \\
\text { and anaesthetic }\end{array}$ \\
\hline 41 & $\begin{array}{l}\text { Datura stramonium } \\
\text { (Solanaceae) }\end{array}$ & Umattai & All parts & $\begin{array}{c}\text { Antimicrobial and Anticancer } \\
\text { activity. }\end{array}$ \\
\hline 42 & $\begin{array}{l}\text { Delonix regia } \\
\text { (Fabaceae) }\end{array}$ & Semmayirkondrai & Seed & $\begin{array}{l}\text { The seed is carminative, purifies } \\
\text { and enriches the blood and is } \\
\text { used in cases of inflammation, } \\
\text { "ear ache" and chest complaint }\end{array}$ \\
\hline 43 & $\begin{array}{l}\text { Desmostachya bipinnata } \\
\text { (Poaceae) }\end{array}$ & Darbai pul & Leaves & $\begin{array}{l}\text { Dysentery and menorrhagia, and } \\
\text { as a diuretic }\end{array}$ \\
\hline 44 & $\begin{array}{l}\text { Eclipta alba } \\
\text { (Asteraceae) }\end{array}$ & Karsalamkanni & Leaf & $\begin{array}{c}\text { Leaf is used for the treatment } \\
\text { of Diabetes }\end{array}$ \\
\hline 45 & $\begin{array}{l}\text { Eclipta procera } \\
\text { (Asteraceae) }\end{array}$ & $\begin{array}{c}\text { Mangel } \\
\text { Karisalankanni }\end{array}$ & Whole parts & $\begin{array}{c}\text { Antibacterial activity and } \\
\text { Jaundice. }\end{array}$ \\
\hline 46 & $\begin{array}{l}\text { Eclipta prostrate } \\
\text { (Asteraceae) }\end{array}$ & $\begin{array}{l}\text { VellaiKarisalankann } \\
\text { i }\end{array}$ & Whole parts & $\begin{array}{c}\text { Antibacterial activity and Eye } \\
\text { diseases. }\end{array}$ \\
\hline 47 & $\begin{array}{l}\text { Enicostem malittorale } \\
\text { (Gentianaceae) }\end{array}$ & Vellaruku & Leaf & $\begin{array}{l}\text { The powered leaves are used } \\
\text { for diabetes. }\end{array}$ \\
\hline 48 & $\begin{array}{l}\text { Ervatamia divaricate } \\
\text { (Apocyanaceae) }\end{array}$ & Nantiyavarttam & Leaf & $\begin{array}{c}\text { Antibacterial and Antifungal } \\
\text { activity. }\end{array}$ \\
\hline 49 & $\begin{array}{l}\text { Erythrina indica Lam. } \\
\text { (Fabaceae) }\end{array}$ & Kalyanamurungai & Leaf & $\begin{array}{l}\text { Leaves relive body pain; } \\
\text { headache, fever, cold, fruits treat } \\
\text { diabetics. }\end{array}$ \\
\hline 50 & $\begin{array}{c}\text { Eucalyptus globules Labill. } \\
\text { (Myrtaceae) }\end{array}$ & Neelagri & Leaf & $\begin{array}{l}\text { Latex is applied on the skin to } \\
\text { alleviate body pain \& never } \\
\text { disorders. }\end{array}$ \\
\hline 51 & $\begin{array}{c}\text { Eugenia jambolana } \\
\text { (Myrtaceae) }\end{array}$ & Naval & Seed & $\begin{array}{l}\text { Early morning seeded powered } \\
\text { is taken to cure diabetes. }\end{array}$ \\
\hline 52 & $\begin{array}{l}\text { Euphorbia heterophylla } \\
\text { (Euphorbiaceae) }\end{array}$ & $\begin{array}{l}\text { Amman } \\
\text { Paccarici }\end{array}$ & Aerial parts & Remove intestinal worms \\
\hline 53 & $\begin{array}{l}\text { Euphorbia hirta } \\
\text { (Euphorbiaceae) }\end{array}$ & Amman pacharisi & Leaf & $\begin{array}{l}\text { Leaf juice is taken orally for } \\
\text { treatment of diabetes }\end{array}$ \\
\hline 54 & $\begin{array}{l}\text { Ficusbenghalensis } \\
\quad \text { (Moraceae) }\end{array}$ & Aalamaram & Bark & $\begin{array}{c}\text { Bark decoction is used for } \\
\text { diabetes }\end{array}$ \\
\hline
\end{tabular}




\begin{tabular}{|c|c|c|c|c|}
\hline 55 & $\begin{array}{l}\text { Ficus racemosa } \\
\text { (Moraceae) }\end{array}$ & Atthi & Root & $\begin{array}{l}\text { Root decoction is taken orally } \\
\text { to cure diabetes. }\end{array}$ \\
\hline 56 & $\begin{array}{l}\text { Ficus religiosa } \\
\text { (Moraceae) }\end{array}$ & Arasu & Leaf & Antibacterial activity. \\
\hline 57 & $\begin{array}{l}\text { Gisekia pharnaceoides } \\
\text { (Aizoaceae) }\end{array}$ & Manalikkirai & Aerial parts & Antibacterial activity. \\
\hline 58 & $\begin{array}{l}\text { Gymnema sylvestre } \\
\text { (Apocyanaceae) }\end{array}$ & Sakkaraikolli & Leaf & Leaf juice is taken daily \\
\hline 59 & $\begin{array}{l}\text { Hemidesmus indicus } \\
\text { (Asclepiadaceae) }\end{array}$ & Nannari & Aerial parts & Fever and Skin diseases \\
\hline 60 & $\begin{array}{l}\text { Heteropogon contortus } \\
\text { (Poaceae) }\end{array}$ & Poonthodapam & Whole plant & $\begin{array}{l}\text { Native Hawaiians used to } \\
\text { construct pili houses. }\end{array}$ \\
\hline 61 & $\begin{array}{l}\text { Hibiscus cannabinus } \\
\text { (Malvaceae) }\end{array}$ & Pulichakeerai & Leaf & Antimicrobial activity. \\
\hline 62 & $\begin{array}{l}\text { Hibiscus rosasinensis } \\
\text { (Malvaceae) }\end{array}$ & Semparuthi & $\begin{array}{l}\text { Leaf } \\
\text { Flower }\end{array}$ & Hair growth and hair infections. \\
\hline 63 & $\begin{array}{c}\text { Jasminum angustifolium } \\
\text { (Oleaceae) }\end{array}$ & Kattu Malligai & $\begin{array}{l}\text { Root and } \\
\text { flower }\end{array}$ & $\begin{array}{l}\text { Skin diseases, ulcers, diseases of } \\
\text { eye, stomatitis, pruritus, anti- } \\
\text { toxic. }\end{array}$ \\
\hline 64 & $\begin{array}{l}\text { Lablab purpureus } \\
\text { (Papilionoideae) }\end{array}$ & Avarai & Leaf & $\begin{array}{c}\text { Alexipharmic, Emmenagogue, } \\
\text { Astringent, diuretic, } \\
\text { anaphrodisiac, stomachic and } \\
\text { antispasmodic. }\end{array}$ \\
\hline 65 & $\begin{array}{l}\text { Lantana camara Linn. } \\
\text { (Verbenaceae) }\end{array}$ & Unni chedi & Leaf & $\begin{array}{l}\text { Leaf juice improves digestion } \\
\text { in children and fruits - treat } \\
\text { diabetics. }\end{array}$ \\
\hline 66 & $\begin{array}{l}\text { Lawsonia inermis } \\
\text { (Lythraceae) }\end{array}$ & Maruthani & Leaf & $\begin{array}{l}\text { Antimicrobial activity and } \\
\text { Cooling of body. Leaves } \\
\text { applied on foot to cure etching } \\
\text { and healing of the crack. }\end{array}$ \\
\hline 67 & $\begin{array}{l}\text { Lucas aspera Sprong. } \\
\text { (Lamiaceae) }\end{array}$ & Thumbai & Leaf & $\begin{array}{l}\text { Leaves used to relive tooth } \\
\text { ache and prevents tooth/gum } \\
\text { infection. }\end{array}$ \\
\hline 69 & $\begin{array}{l}\text { Millettia pinnata } \\
\text { (Fabaceae) }\end{array}$ & Pungamaram & Flowers & anti-diabetic action \\
\hline 70 & $\begin{array}{l}\text { Mimosa pudica } \\
\text { (Mimosaceae) }\end{array}$ & Thottasurungi & Leaf & Wounds \\
\hline 71 & $\begin{array}{l}\text { Mollugo cerviana } \\
\text { (Aizoaceae) }\end{array}$ & Porpadakam & Aerial part & Treat fever. \\
\hline 72 & $\begin{array}{c}\text { Morinda coreia } \\
\text { (Rubiaceae) }\end{array}$ & Nuna & Leaf & Antibacterial activity. \\
\hline 73 & $\begin{array}{l}\text { Nerium oleander (sol) } \\
\text { (Apocynaceae) }\end{array}$ & Arali & Fruit Edible & Ear pain \\
\hline 74 & $\begin{array}{l}\text { Ocimum basilicum } \\
\text { (Labiatae) }\end{array}$ & Karpura Thulasi & Leaf & $\begin{array}{l}\text { Leaf juice is mixed with cumin } \\
\text { is given to cure the ear pains. }\end{array}$ \\
\hline 75 & $\begin{array}{l}\text { Ocimum sanctum } \\
\quad \text { (Labiatae) }\end{array}$ & Thulasi & Leaf & $\begin{array}{l}\text { Leaf juice is mixed with cumin } \\
\text { is given to cure the dry cough. }\end{array}$ \\
\hline 76 & $\begin{array}{l}\text { Oxalis corniculata } \\
\text { (Oxalidaceae) }\end{array}$ & Pulichchakeerai & Leaf & Antimicrobial activity. \\
\hline
\end{tabular}




\begin{tabular}{|c|c|c|c|c|}
\hline 77 & $\begin{array}{l}\text { Phyllanthus amaru } \\
\text { (Euphorbiaceae) }\end{array}$ & Kilanelli & Leaf & $\begin{array}{l}\text { Leaf juice is taken orally to } \\
\text { treat diabetes. }\end{array}$ \\
\hline 78 & $\begin{array}{l}\text { Polyalthia longifolia } \\
\text { (Annonaceae) }\end{array}$ & Nettilinkam & Leaf & $\begin{array}{l}\text { Fever, gonorrhea, uterus } \\
\text { aliment, leucorrhoea, mouth } \\
\text { ulcer heart problem, blood } \\
\text { pressure and stimulated } \\
\text { respiration. }\end{array}$ \\
\hline 79 & $\begin{array}{l}\text { Pongamia pinnata } \\
\text { (Fabaceae) }\end{array}$ & Pungam & $\begin{array}{l}\text { Whole Plant } \\
\text { Extract }\end{array}$ & Antiseptic \\
\hline 80 & $\begin{array}{l}\text { Psidium guajava } \\
\text { (Myrtaceae) }\end{array}$ & Koiyaa & Fruit & $\begin{array}{l}\text { Daily one fruits is taken to } \\
\text { cure diabetes. }\end{array}$ \\
\hline 81 & $\begin{array}{c}\text { Solanum nigrum } \\
\text { (Solanaceae) }\end{array}$ & Manathakalli & Aerial parts & Antimicrobial activity. \\
\hline 82 & $\begin{array}{l}\text { Solanum virginianum } \\
\text { (Solanaceae) }\end{array}$ & Kandankatthiri & Fruits & cough, asthma and chest pain \\
\hline 83 & $\begin{array}{l}\text { Spermacocehispida } \\
\text { (Rubiaceae) }\end{array}$ & Nathachuri & Leaf & $\begin{array}{c}\text { The powered leaves are taken } \\
\text { twice daily. }\end{array}$ \\
\hline 84 & $\begin{array}{l}\text { Thespesia populnea } \\
\text { (Malvaceae) }\end{array}$ & Puvaracu & Leaf & Skin disease \\
\hline 85 & $\begin{array}{l}\text { Tridax procumbens } \\
\text { (Rubiaceae) }\end{array}$ & $\begin{array}{l}\text { Kenatrupasan } \\
\text { Vettukaya puntu }\end{array}$ & Leaf & $\begin{array}{l}\text { Leaf juice is applied externally } \\
\text { for healing wounds. }\end{array}$ \\
\hline 86 & $\begin{array}{l}\text { Vitex negundo } \\
\text { (Verbanaceae) }\end{array}$ & Nochi & Leaf & Headache and Sinus problem. \\
\hline 87 & $\begin{array}{l}\text { Withania somnifera } \\
\quad \text { (Solanaceae) }\end{array}$ & Ashwagandha & All parts & $\begin{array}{l}\text { Antimicrobial activity and Leaf } \\
\text { paste in cow's milk used to treat } \\
\text { asthma. }\end{array}$ \\
\hline 88 & $\begin{array}{l}\text { Ziziphus mauritiana } \\
\text { (Rhamnaceae) }\end{array}$ & Elandai & Fruits & Nutritious and rich in vitamin $\mathrm{C}$. \\
\hline
\end{tabular}

The survey reveals that Mahadevan hill is enriched with many plants. The medicinal plants in the hill are still used by the local inhabitants for many purposes. The biodiversity of the hill should be further explored and conserved in future. Through this ethnobotanical survey, the obtainability and existence of many medicinal plants have been examined and verified.

We observed that these plants are be used as drugs by pharmacologically unexplored areas of India, which may be utilized for the better human health. This study offers a ideal for studying the association between plants and people, within the context of traditional therapies. This study also gathered a broad scale of information regarding medicinal plants used by the peoples of Vellore district.
The survey specified that, the study area has outstanding plant diversity with sufficiently of medicinal plants to treat a wide range of human ailments. It is evident from the interviews conducted in Mahadevan hill.

Knowledge of medicinal plants is limited to traditional healers, herbalists who are living in rural areas and collecting the medicinal plants from the Mahadevan hill unscientifically. It is concluded that the unscientific collection of ethnobotanical plants from the Mahadevan hill of Vellore district possess greater pressure on the depletion of diversity of the local region. Hence, there is an crucial need to assess the biodiversity of the local forest, and conserve the biodiversity as well as the traditional knowledge by appropriate documentation and conservation strategies. 


\section{Acknowledgements}

The authors thank the management of Islamiah College (Autonomous) for providing the facilities to carry out this research work.

\section{References}

Davidson-Hunt I . Ecological ethnobotany : stumbling toward new practices and paradigms. MASA J. 2000;16:1-13.

Fabricant L, Farnsworth NR. The value of plants used in traditional medicine for drug discovery. Environmental HealthPerspectives. 2001; 109: 69 - 75.

Kolanjinathan K, Saranraj P. Pharmacological activity of Mangrove medicinal plants against pathogenic bacteria and fungi. Academic Discourse: An International Journal. 2015; 8(1): $1-15$.

Mohamed Tariq NPM, Md. Rayees Ifham S. Ethnobotanical Survey of Medicinal Plants in Yelagiri Hills of Tamil Nadu. Res. J. Pharm. Technol. 2013; 6 (6): 652-654.

Mukherjee PK, Wahile A. Integrated approaches towards drug development from Ayurveda and other Indian system of medicines. Journal of Ethnopharmacolology. 2006; 103:25.
Ethnobotanical survey of medicinal plants from Vellore district, Tamil nadu, India. International Journal of Advanced Research in Biological Sciences. 2016; 3(9): 238-246.

Parinitha M, Srinivasa BH, Shivanna MB. Medicinal plant wealth of local communities in some villages in Shimoga Distinct of Karnataka, India. Journal of Ethnopharmacolology. 2005; 98:307-312.

Perumal Samy R, Ignacimuthu S. Journal of Ethnopharmacolology. 1008; 62: 173.

Saranraj P, Sujitha D. Mangrove Medicinal Plants: A Review. American EurasianJournal of Toxicological Sciences. 2015; 7(3): $146-156$.

Sastri K, Chaturvedi GN, Charak Drdhbala. The Charak Samhita. 22 revised edition. Edited by Sastri R, Uppadhayaya Y, Pandeya GS, Gupta B, Mishra B. Chukhamba Bharti Acedemy, Varansi. 1996.

Toledo BA, Galetto L Colantonio S. Ethnobotanical knowledge in rural communities of Cordoba (Argentina): the importance of cultural and biogeographical factors. Journal of Ethno biology and Ethnomedicine. 2009; 5:40.

Saranraj P, Bhavani L, Suganthi K.

\section{How to cite this article:}

Devendiran. D, H. Abdul Jaffar Ali, K. Muhammed Shariq and Farook. M.A. 2020. Survey of Medicinal Plants in Mahadevan Hill, K.V. Kuppam, At Vellore District, Tamil Nadu. Int.J.Curr.Microbiol.App.Sci. 9(03): 969-977. doi: https://doi.org/10.20546/ijcmas.2020.903.114 